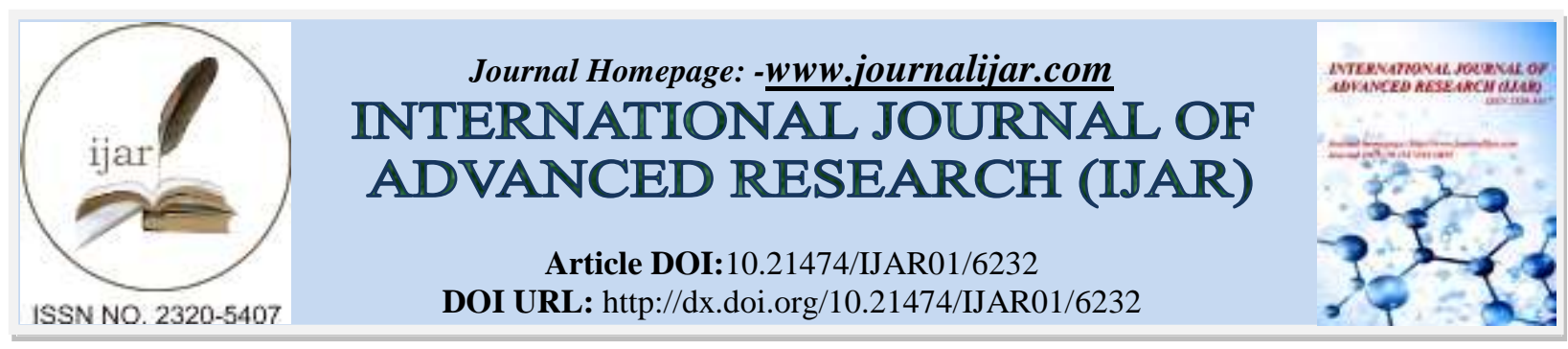

RESEARCH ARTICLE

\title{
A RARE CASE REPORT: MACRODYSTROPHIA LIPOMATOSA.
}

Kirtana Shah ${ }^{1}$, Mahesh Pukar ${ }^{2}$ and Anupam Shukla ${ }^{1}$.

1. Resident, Department of General Surgery, SBKSMI \& Research Centre, Vadodara, Gujarat, India.

2. Professor, Department of General Surgery, SBKSMI \& Research Centre, Vadodara, Gujarat, India.

\section{Manuscript Info}

Manuscript History

Received: 06 November 2017

Final Accepted: 08 December 2017

Published: January 2018

Key words:-

Macrodystrophialipomatosa,

macrodactyly.

\section{Abstract}

Macrodystrophialipomatosa is a rare cause of congenital macrodactyly, characterized by progressive proliferation of all mesenchymal elements, with a disproportionate increase in fibroadipose tissue. This developmental anomaly is reportedly more common in the foot than in the hand, with a predilection for the plantar and median nerve distribution. We present a case of MDL of the foot. Preoperative diagnosis was however made on the basis of radiography and MRI and was later confirmed on surgery

Copy Right, IJAR, 2018,. All rights reserved.

\section{Introduction:-}

Macrodystrophialipomatosa (ML) is a rare non-hereditary congenital developmental anomaly causing disproportionate overgrowth of one or several digits. It may cause a localised gigantism or involve the entire limb. It is characterised by progressive hamartomatous enlargement of the fibro-fatty tissue and should be differentiated from macromelia or hemihypertrophy. We present a case of this anomaly limited to the unilateral left foot involving great toe and second toe.

\section{Case Report:-}

A 24-year-old male presented to our institute with a history of enlargement of left great toe and second toe since childhood (Figure 1). He did not offer any other complaints. There was no history of similar complaints or neurofibromatosis in his family members suggestive of non-hereditary nature of the lesion. There were no obvious cutaneous vascular lesions. There was no neurological deficit. The other digits and contralateral foot were normal. India. 


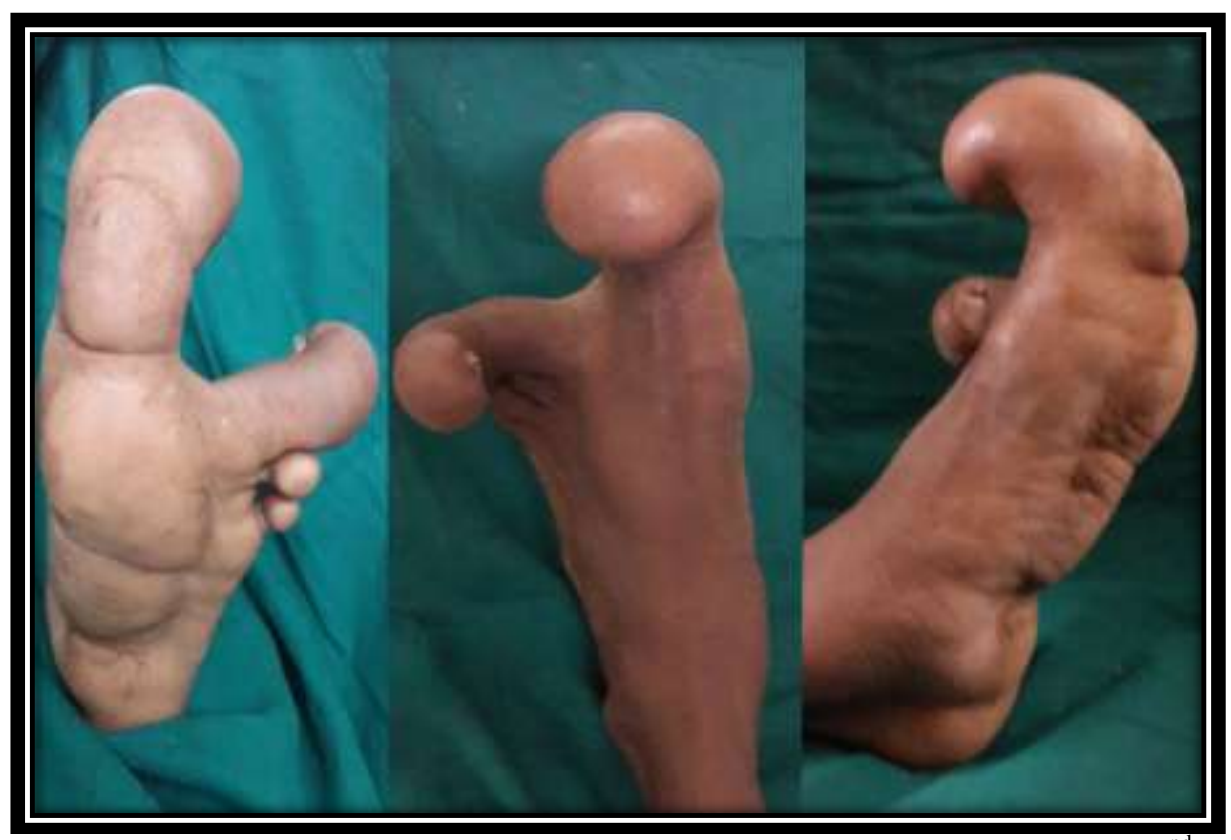

Radiographs of the left foot showed gross increase in the girth of soft tissue of great toe and the $2^{\text {nd }}$ toe as compared to the contralateral side. There was also a deformity of the distal phalanges of the great and $2^{\text {nd }}$ toe with early osteoarthritic changes in $1^{\text {st }}$ and $2^{\text {nd }}$ metatarsophalangeal joints. The phalanges and metatarsals were enlarged as compared to contralateral side.

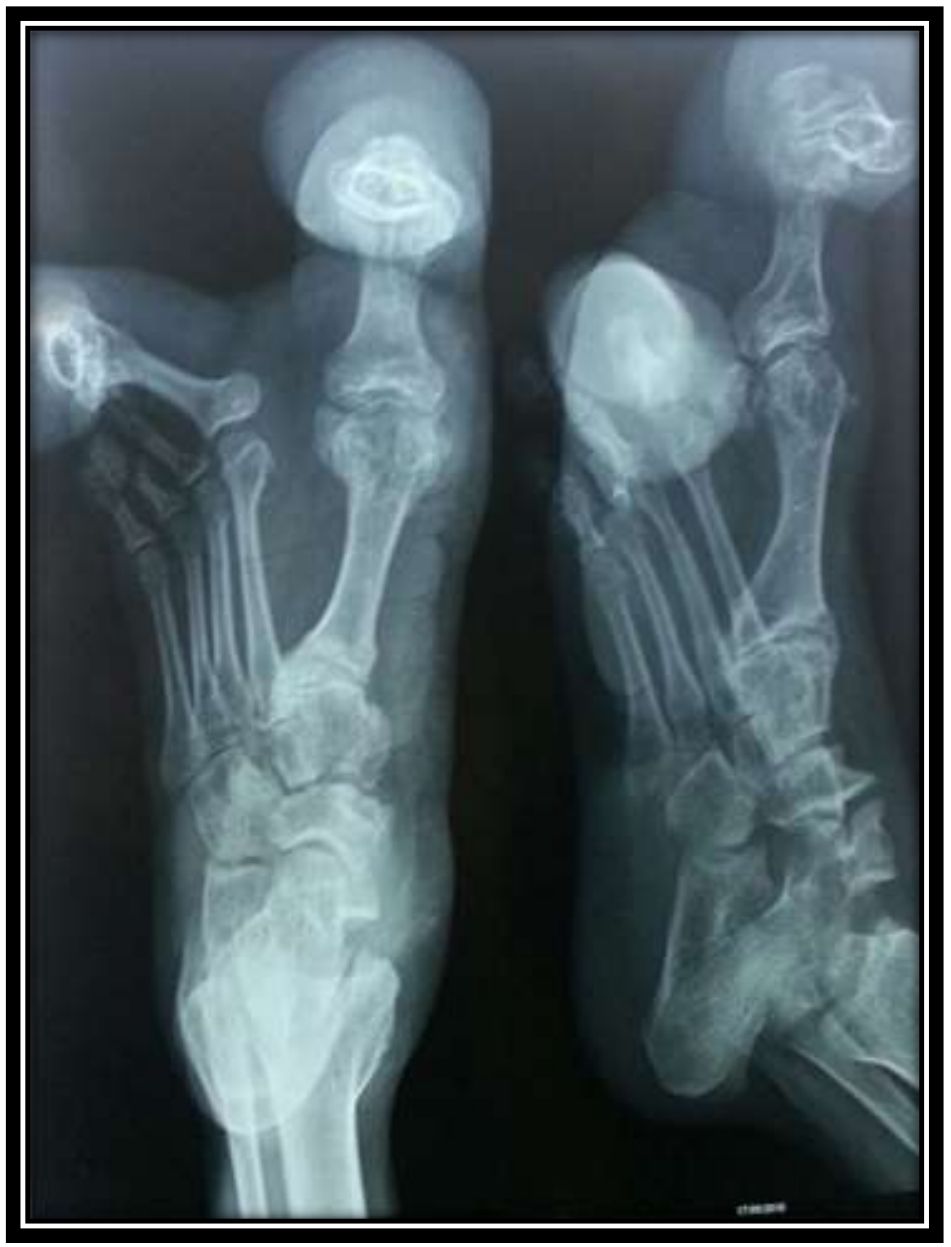


MRI revealed diffused thickening of soft tissue appearing heterogeneously hyperintense with multiple thin linear low-signal intensity areas consistent with fibrous strands. There was also excess of unencapsulated fibro-adipose tissue.

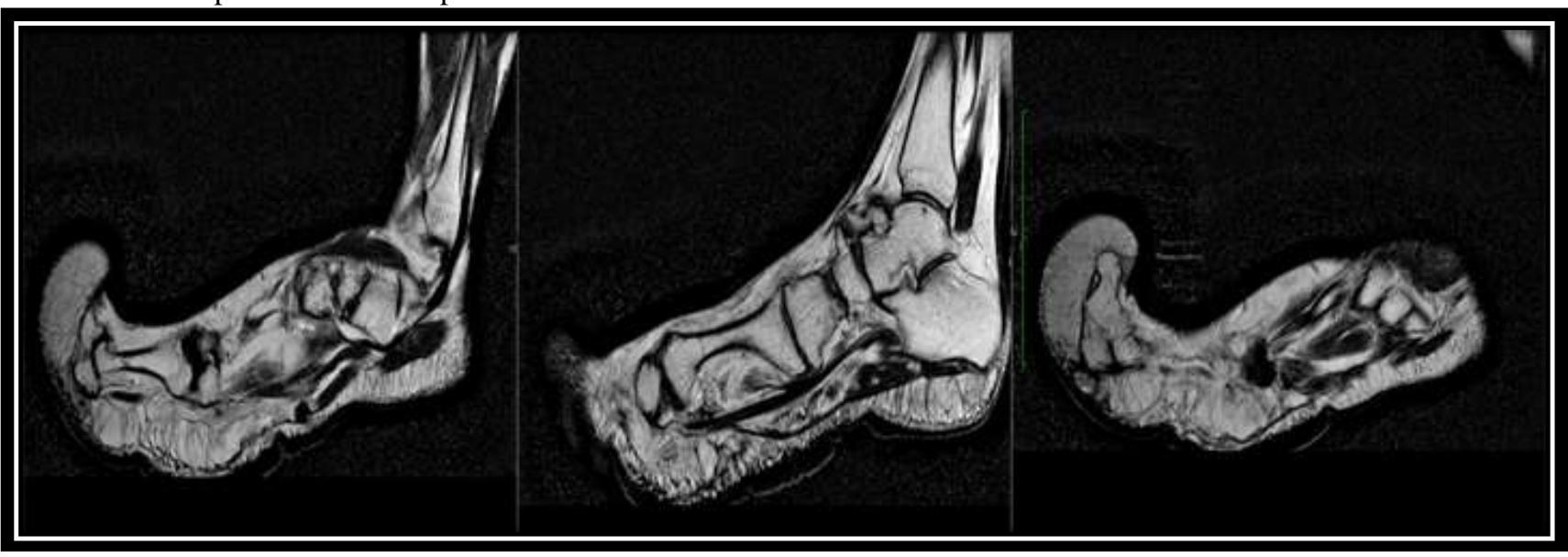

\section{Operative notes:-}

After getting the pre anaesthetic work up done patient was taken under spinal anaesthesia. Supine position was given and painting and draping done. Following which a fishmouth incision was given for traansmetatarsal amputation. This revealed the marked hypertrophy of fat along with adhesions in between the skin and periosteum. Transmetatarsal amputation was done. Hypertrophied metatarsal bones were cut from the neck and myoplasty was performed between dorsal and plantar compartments. Debulking of the remaining compartment was done. Hemostasis was achieved. Patient tolerated the procedure well.

The patient underwent surgery for cosmetic reasons.

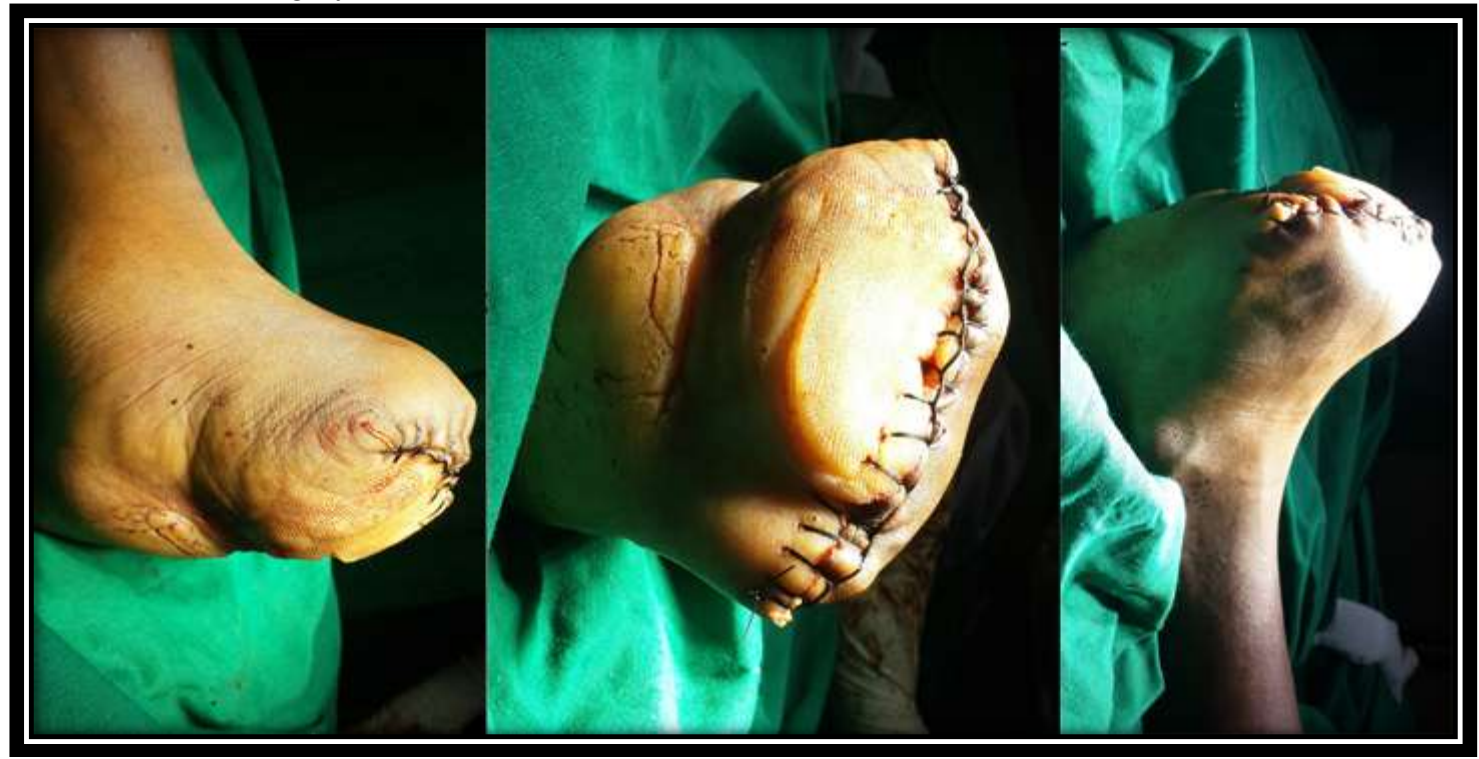

\section{Post-operative notes:-}

The patient was mobilized on post-operative day 2. Check dressing as well as catheter removal was done on postoperative day 3 . The suture line was clean and healthy.

Regular physiotherapy was done. Histopathology showed presence of adipose tissue in the excised specimen scattered in a fine mesh-like fibrous tissue extending to involve the periosteum. Patient was diagnosed as macrodystrophialipomatosa. On post-operative day 10 there was wound gapping following which dressing was done 
daily. Inspite this he underwent skin tissue grafting on post-operative day 25 . There was $100 \%$ uptake of the graft. Patient was discharged on postoperative day 32. Orthotic supplements were given to the patient on follow up.

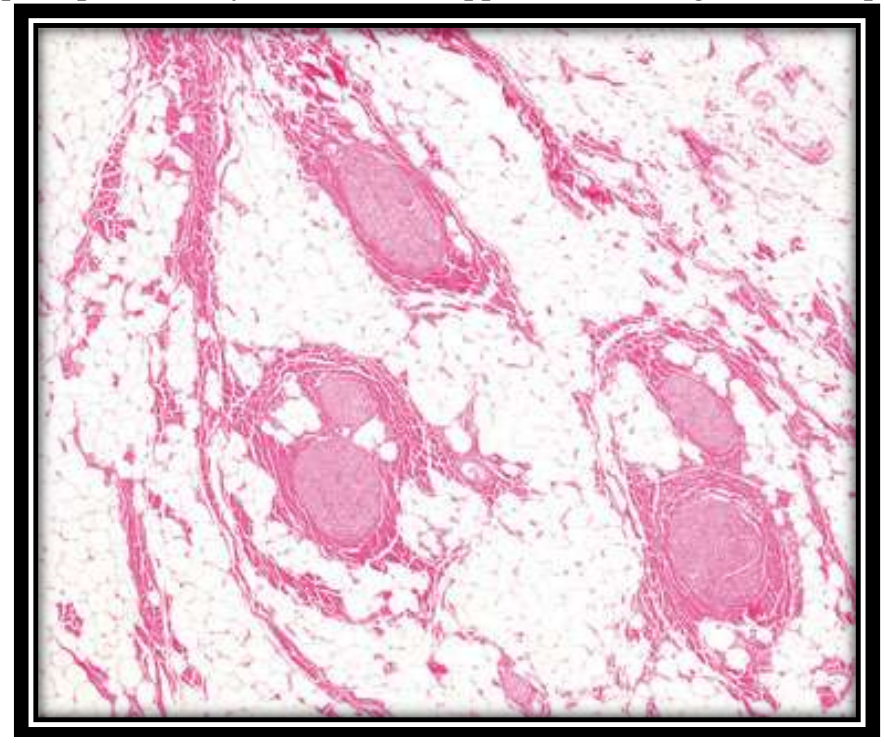

\section{Discussion:-}

- Macrodystrophialipomatosa is a rare form of localised gigantism characterised by progressive overgrowth of all the mesenchymal elements with a disproportionate increase in the fibroadipose tissues.

- Occurs most frequently in the distribution of the median nerve in the upper extremity and in the distribution of the plantar nerves in the lower extremity.

- In 1925, Feriz was the first person to use this term for localised gigantism of the lower extremity. Barsky on the other hand gave a more detailed description of the disease involving local gigantism of digits. He described two forms of true macrodactyly. In the first or the static form, the size of the enlarged digits increases proportionally in relation to the rest of body. In the second or the progressive form which is less common, the growth rate of the enlarged digits is disproportionate to the growth of rest of the body. The progressive form may be associated with fatty overgrowth, and it is similar to what Feriz described.

- Our case would fit into the first category.

- This disease is non-hereditary in nature and has a slight male preponderance as in our case. The lower limb is involved more commonly. The region supplied by medial plantar nerve, that is, first to third toes, is frequently involved and the fifth digit is the rarest to be involved. ML commonly involves unilateral digits but rarely, a bilateral involvement has been described in literature.

- It is usually recognised in the neonatal period but starts to cause problems as the child grows.

- There may be difficulty in walking though, more often than not, a surgical consultation is sought for cosmetic rather than mechanical reasons.

- As the child grows there may be degenerative changes of small joints and compression of the neurovascular structure. Patient may present with features of carpal tunnel syndrome due to involvement of median nerve. The growth of bones normally ceases by puberty. Histopathology shows abundant increase in the adipose tissue scattered in a fine mesh-like fibrous tissue. Besides affecting subcutaneous tissue it can involve underlying nerve sheaths, muscles, periosteum, and bone marrow. Exact aetiopathogenesis is obscure.

- The proposed mechanisms include lipomatous degeneration, disturbed foetal circulation, disturbances of growth inhibiting factors, an error in segmentation, and trophic influence of tumefied nerve. The pathogenesis of bony enlargement is thought to be because of endosteal and periosteal deposition of bone.

- Plain radiographs may demonstrate abnormalities in both the soft tissues and bony elements. The soft tissue overgrowth is most marked on the volar aspect. This volar overgrowth results in dorsal deviation of the digits.The affected phalanges and metatarsal bones show an increase in width and length, and often splayed at their distal ends. The articular surfaces may slant and in late childhood severe secondary degenerative changes may affect the joints. All these features were present in our case. Characteristic radiolucencies seen are indicative of the fatty nature of the soft tissue.

- Presence of syndactyly, polydactyly,and clinodactyly has been reported. 
- Computed tomography of the involved extremities shows excessive fatty proliferation. Fatty infiltrations of the involved muscles with separation of the muscle fibres are also often evident.

- Magnetic resonance imaging characteristically shows abundant adipose tissues in the involved areas. This fat has the same signal intensity as the normal subcutaneous fat. But contrary to lipoma, the abnormal fat in this disorder is not encapsulated. Linear hypointense bands may be noted within this redundant adipose tissue representing fibrous strands. Fatty infiltration of the involved muscles as well as, the bony overgrowth and cortical thickening of the enlarged bones can be well demonstrated on the MR imaging.

- Histopathology in our case proved the diagnosis.

- Differential diagnosis to localised gigantism of the digit includes congenital localised gigantism such as fibrolipomatoushamartoma of a nerve, neurofibromatosis, haemangiomatosis, lymphangiomatosis, Proteus syndrome and Klippel-Trenaunay-Weber syndrome that includes macrodactyly with haemangiomas. But in the absence of family history and any cutaneous or systemic manifestation these diagnoses are unlikely.

- Patients seek medical help more often for cosmetic reasons rather than for mechanical difficulties which is normally late in life. Surgical correction is the treatment of choice for ML. Our patient sought surgical intervention as he believed this disability would have made him unfit for recruitment in the defence forces.

- The main surgical principle in treating this lesion is to improve cosmetic appearance and preserve neurologic function as far as possible and this is usually done after puberty when growth ceases.

- Judicious and planned use of multiple debulking procedures, epiphysiodesis, and various osteotomies are advised to achieve the best results.

- Complications associated with overzealous debulking procedure lead to nerve injury with an incidence reported as high as $30-50 \%$. A localised recurrence rate of 33-60\% makes the management of ML more demanding.

\section{Conclusion:-}

In conclusion, ML is a rare form of congenital localised gigantism, and surgical consultation is often sought for cosmetic reasons. Imaging provides vital clues to an accurate diagnosis which is confirmed by histopathology. Surgery can be offered to patients after puberty for cosmetic as well as functional problems

\section{References:-}

1. Sudesh P, Raj N, Kumar R and Parakash S: Macrodystrophialipomatosa. Foot (Edinb) 22: 172-174, 2012.

2. Gaur AK, Mhambre AS, Popalwar H and Sharma R: Macrodystrophialipomatosa of foot involving great toe. Foot (Edinb) 24: 86-88, 2014.

3. Singla V, Virmani V, Tuli P, et al: Case Report: Macrodystrophialipomatosa-Illustration of two cases. Indian J Radiol Imaging 18: 298-301, 2008.

4. Selcen O, Baykara M, Öztürk M and Özyazgan İ: Macrodactyly: Radiologic findings of a rare anomaly. Erciyes Medical Journal 26: 44-47, 2004.

5. Watt AJ and Chung KC: Macrodystrophialipomatosa: a reconstructive approach to gigantism of the foot. J Foot Ankle Surg 43: 51-55, 2004.

6. Wang YC, Jeng CM, Marcantonio DR and Resnick D: Macrodystrophialipomatosa. MR imaging in three patients. Clin Imaging 21: 323-327, 1997.

7. Barsky AJ: Macrodactyly. J Bone Joint Surg Am 49: 1255-1266, 1967. 